\title{
Influence of Antithrombotic Medication on the Risk of Chronic Subdural Hematoma Recurrence after Burr-Hole Surgery
}

\author{
Jongwook Choi, Jinsu Pyen, Sungmin Cho, Jongyeon Kim, Younmoo Koo, Kum Whang \\ Department of Neurosurgery, Wonju Severance Christian Hospital, Yonsei University Wonju College of Medicine, Wonju, Korea
}

Objective : Chronic subdural hematoma (CSDH) is one of the most common intracranial hemorrhages. It can be managed with a simple surgical treatment such as burr-hole trephination and drainage. However, it has a relatively high recurrence rate. The mechanisms and risk factors for the recurrence have not yet been clearly identified and studies have reported varying results.

Methods : We analyzed 230 patients with CSDH who were treated with burr-hole trephination and drainage at our institution from March 2011 to March 2016. The patients were divided into recurrence and non-recurrence groups and the medical records of each group were used to analyze the risk factors associated with CSDH recurrence.

Results : After burr-hole trephination and drainage, CSDH recurrence was observed in 49 (21.3\%) of the 230 patients. In univariate analysis, none of the factors showed statistical significance with respect to CSDH recurrence. In multivariate analysis, preoperative antithrombotic medication was the only independent risk factor for CSDH recurrence (odds ratio, 2.407; 95\% confidence interval, 1.047-5.531).

Conclusion : The present study found that preoperative antithrombotic medication was independently associated with CSDH recurrence.

Key Words : Hematoma, Subdural, Chronic · Recurrence · Anticoagulants · Platelet aggregation inhibitors.

\section{INTRODUCTION}

Chronic subdural hematoma (CSDH) is one of the most common intracranial hemorrhages, but the pathogenic mechanism of its occurrence is not yet clear. With an increase in the elderly population, the number of CSDH patients is increasing, with 1-13.5 global incidences per 100000 of the popula- tion $^{9,10)}$. In persons aged 65 years and older, a high incidence of CSDH (60-80 per 100000 of the population) is observed ${ }^{13)}$. The treatment of CSDH is relatively easy, which primarily consists of simple burr-hole trephination and drainage. The prognosis is good, but the recurrence rate is reported to be relatively high $(2-38 \%)^{10,11,13}$. Although much research has been conducted on the relatively high recurrence rates, the results

- Received : September 20, 2019 •Revised : November 16, $2019 \bullet$ Accepted : December 31, 2019

- Address for reprints : Kum Whang

Department of Neurosurgery, Wonju Severance Christian Hospital, Yonsei University Wonju College of Medicine, 20 Ilsan-ro, Wonju 26426, Korea Tel : +82-33-741-0593, Fax : +82-33-746-2287, E-mail : whangkum@yonsei.ac.kr, ORCID : https://orcid.org/0000-0002-3701-847X 
of the studies on the mechanism and the risk factors remain controversial.

The purpose of the present study was to analyze the association between the use of antiplatelet or anticoagulant medication and CSDH recurrence in patients at a single institution.

\section{MATERIALS AND METHODS}

\section{Selection of patients and data collection}

The present retrospective analysis was approved by the Institutional Review Board of Wonju Severance Christian Hospital (CR319108). A total of $286 \mathrm{CSDH}$ patients who were surgically treated between March 2011 and March 2016 were studied retrospectively. Thirty-six patients were lost to followup, 11 patients had insufficient medical records, and 11 patients were subjected to reoperation due to insufficient drainage of the initial CSDH. Thus, 56 patients were excluded from the study. The remaining $230 \mathrm{CSDH}$ patients who underwent surgical treatment were enrolled in this study. These patients were divided into two groups : the recurrence group and the non-recurrence group. Each group was analyzed for past health history (hypertension, diabetes mellitus, chronic alcoholism, and use of antithrombotic agents).

\section{Surgical procedure and management}

All patients underwent one or two burr-hole trephination surgeries with closed drainage under general anesthesia. After dural incision and hemostasis, the outer hematoma membrane was opened. A drainage catheter was inserted into the hematoma cavity and connected to a closed drainage system. All patients were maintained in supine position and supplied with sufficient fluid to promote brain expansion. Three days after the surgery, the catheter was removed after confirming the reduction of the hematoma through brain computed tomography scan.

\section{Statistical analysis}

All variables were evaluated for association with CSDH recurrence using the two-sample t-test or the chi-squared test. Logistic regression analysis was used to identify the independent risk factors to predict $\mathrm{CSDH}$ recurrence and the results were expressed as odds ratio (OR) with 95\% confidence interval (CI). Statistical significance was accepted for $p$ values
$<0.05$, and the analysis was conducted using SPSS ver. 24.0 (IBM, Armonk, NY, USA).

\section{RESULTS}

Of the total 230 patients who underwent the CSDH surgery, $164(71.3 \%)$ were male. The mean age was $69.4 \pm 13.1$ years. The patients' history included hypertension in 119 patients (51.7\%), diabetes mellitus in 44 patients (19.1\%), chronic alcoholism in 34 patients (14.8\%), and history of antithrombotic medication in 36 patients (15.7\%). The recurrence rate was 21.3\% (Table 1).

History of antithrombotic medication showed a greater correlation with $\mathrm{CSDH}$ recurrence when compared with the nonrecurrence group (24.5\% vs. $13.3 \%)$. However, the results were not statistically significant $(p=0.055)$. Other variables showed no statistically significant correlation with recurrence of $\mathrm{CSDH}$ in the univariate analysis (Table 2). Binary logistic regression was performed to identify the independent risk factors related to $\mathrm{CSDH}$ recurrence. Preoperative antithrombotic medication was observed to be the only independent variable related to $\mathrm{CSDH}$ recurrence (OR, 2.41; 95\% CI, 1.05-5.53). However, when categorizing antithrombotic medications into

Table 1. Characteristics of 230 patients who underwent burr-hole surgery for chronic subdural hematoma

\begin{tabular}{lc}
\hline Characteristic & Value (n=230) \\
\hline Gender, male & $164(71.3)$ \\
Age & $69.4 \pm 13.1$ \\
History & \\
Hypertension & $119(51.7)$ \\
Diabetes mellitus & $44(19.1)$ \\
Antithrombotic medication & $36(15.7)$ \\
$\quad$ Antiplatelets medication & $30(13.0)$ \\
$\quad$ Anticoagulants medication & $6(2.6)$ \\
Chronic alcoholism & $34(14.8)$ \\
Bilateral lesion & $86(37.4)$ \\
Preoperative GCS score & $171(74.3)$ \\
15-14 & $36(15.7)$ \\
13-9 & $23(10.0)$ \\
8-3 & $49(21.3)$ \\
\hline Recurrence & \\
\hline Values are presented as mean \pm standard deviation or number (\%). GCS: \\
Glasgow coma scale
\end{tabular}


antiplatelets and anticoagulants, preoperative antiplatelet medication (OR, 2.25; 95\% CI, 0.93-5.47) and preoperative anticoagulant medication (OR, 2.38; 95\% CI, 0.39-14.41), respectively, were not associated with CSDH recurrence (Table 3).

\section{DISCUSSION}

$\mathrm{CSDH}$ is known to be caused by damage to bridging veins after direct or indirect trauma to the brain ${ }^{5}$. There are many prognostic factors related to various structural changes such as decreased intracranial pressure, brain atrophy, changes in the skull, and cerebrospinal fluid fistula ${ }^{12,15)}$. Moreover, nontraumatic factors such as hematologic coagulation pathology, clinical history of chronic alcoholism, arteriovenous malformation, anticoagulant therapy, and bleeding tendency have been reported to aggravate the clinical outcome of $\mathrm{CSDH}^{4,5}$. However, risk factors for CSDH recurrence are inconsistent

Table 2. Comparison between the two groups according to patient characteristics and recurrence of chronic subdural hematoma

\begin{tabular}{|c|c|c|c|}
\hline & $\begin{array}{l}\text { No recurrence } \\
\quad(n=181)\end{array}$ & $\begin{array}{l}\text { Recurrence } \\
(n=49)\end{array}$ & $p$-value \\
\hline Gender, male & $128(70.7)$ & $36(73.5)$ & 0.706 \\
\hline Age & $69.5 \pm 12.6$ & $69.0 \pm 15.0$ & 0.797 \\
\hline \multicolumn{4}{|l|}{ History } \\
\hline Hypertension & $94(51.9)$ & $25(51.0)$ & 0.910 \\
\hline Diabetes mellitus & $37(20.4)$ & $7(14.3)$ & 0.331 \\
\hline $\begin{array}{l}\text { Antithrombotic } \\
\text { medication }\end{array}$ & $24(13.3)$ & $12(24.5)$ & 0.055 \\
\hline $\begin{array}{l}\text { Antiplatelet } \\
\text { medication }\end{array}$ & $20(11.0)$ & $10(20.4)$ & 0.084 \\
\hline $\begin{array}{c}\text { Anticoagulant } \\
\text { medicaiton }\end{array}$ & $4(2.2)$ & $2(4.1)$ & 0.610 \\
\hline Chronic alcoholism & $28(15.5)$ & $6(12.2)$ & 0.573 \\
\hline
\end{tabular}

Values are presented as mean \pm standard deviation or number (\%) according to various studies reported thus far. To date, many studies have been conducted and it is very important to evaluate them.

The use of antithrombotic agents is increasing due to the increase in aging population and development of diagnostic techniques and medical technology. In addition to the benefits of the antithrombotic agents, many researches have been conducted on their side effects. Particularly, the risk of intracranial hemorrhage posed by antithrombotic agents has been studied extensively. However, the treatment guidelines and the plans to minimize the risk remain controversial.

In a meta-analysis related to the prognosis of isolated acute subdural hemorrhage in patients who received antithrombotic therapy, Won et al. ${ }^{26)}$ concluded that patients who received oral anticoagulant therapy showed unfavorable outcome despite successful hemostasis. The thrombocyte inhibitor subgroup of the oral anticoagulants showed a high risk of rebleeding and a high mortality rate. In addition, the direct oral anticoagulants (DOACs) showed unfavorable outcomes and high mortality. Since these antithrombotic agents are consumed for a chronic disease or a comorbidity, they are often resumed after hemostasis is achieved in a case of hemorrhage. Therefore, the effects of antithrombotic medication cannot be ignored in cases of chronic hemorrhage such as $\mathrm{CSDH}$ as well as in acute hemorrhage.

Regarding the pathogenesis of CSDH, it is hypothesized that micro-hemorrhage in a hematoma cavity is associated with recurrence. Antithrombotic agents such as antiplatelet medications and anticoagulants are considered to interfere with the formation of clots in these micro-hemorrhages ${ }^{13)}$. This mechanism is probably associated with CSDH recurrence and the results of this study support this mechanism.

Recent systematic reviews and meta-analyses have reported various conclusions regarding the risk of CSDH recurrence in patients consuming antithrombotic agents at the time of CSDH diagnosis ${ }^{14,18,19)}$. In a systematic review of the use of an-

Table 3. ORs of chronic subdural hematoma recurrence for preoperative antithrombotic medications

\begin{tabular}{lccc}
\hline & Crude OR (95\% Cl) & Adjusted OR* (95\% Cl) & Adjusted OR $^{\dagger}$ (95\% Cl) \\
\hline Antithrombotic medication & $2.12(0.97-4.63)$ & $2.26(1.01-5.06)$ & $2.41(1.05-5.53)$ \\
Antiplatelet medication & $2.06(0.90-4.76)$ & $2.16(0.91-5.11)$ & $2.25(0.93-5.47)$ \\
Anticoagulant medication & $1.88(0.34-10.60)$ & $2.09(0.36-12.16)$ & $2.38(0.39-14.41)$ \\
\hline
\end{tabular}

${ }^{*}$ Adjusted for age, gender. ${ }^{\dagger}$ Adjusted for age, gender, hypertension, diabetes mellitus, chronic alcoholism. OR : odds ratio, $\mathrm{Cl}$ : confidence interval 
tiplatelet medications and anticoagulants in CSDH patients, Nathan et al. ${ }^{14)}$ concluded that anticoagulants were associated with increased risk of rebleeding of $\mathrm{CSDH}$, whereas antiplatelet medications were not. On the contrary, through a systematic review and meta-analysis of the relationship between postoperative recurrence and antithrombotic agent use in patients with CSDH, Poon and Al-Shahi Salman ${ }^{18)}$ concluded that the use of antithrombotic agents at the time of diagnosis of $\mathrm{CSDH}$ may be related to $\mathrm{CSDH}$ recurrence after the surgery (relative risk, 1.38; 95\% CI, 1.00-1.91). Wang et al. ${ }^{23)}$ also concluded that postoperative CSDH recurrence was associated with both anticoagulants (OR, 2.20; 95\% CI, 1.45-3.33) and antiplatelet medications (OR, 1.64; 95\% CI, 1.17-2.30). Wang et al. ${ }^{22)}$ reported that $\mathrm{CSDH}$ recurrence was related to anticoagulants (OR, 1.41; 95\% CI, 1.10-1.81) and antiplatelet medications (OR, 1.23; 95\% CI, 1.01-1.49), and the antithrombotic agents including both anticoagulants and antiplatelet medications increased the risk of $\mathrm{CSDH}$ recurrence, necessitating additional surgery (OR, 1.30; 95\% CI, 1.11-1.52).

Many individual studies have also been conducted regarding the use of antithrombotic agents and $\mathrm{CSDH}$ recurrence. Aspegren et al. ${ }^{2)}$ argued that the recurrence was not affected by anticoagulant and/or antiplatelet aggregation agent therapy prior to the diagnosis of CSDH. Fornebo et al. ${ }^{7)}$ reported no difference in the recurrence rate and mortality between patients who underwent antithrombotic therapy at the time of CSDH diagnosis and those who did not. However, morbidity was higher in the antithrombotic therapy group and early resumption of antithrombotic agents was not associated with recurrence in these patients, rather late resumption of antithrombotic agents increased thromboembolic frequency ${ }^{7}$. Motoie et al. ${ }^{13)}$ reported that antithrombotic agents such as aspirin and warfarin did not increase the CSDH recurrence.

Many studies have reported conflicting results on antithrombotic agents as a risk factor associated with CSDH recurrence. These inconsistent conclusions are believed to result from a variety of study designs for each CSDH study. This implies the need for a systematic research based on a consistent research design in the future.

In addition to the use of antithrombotic agents at the time of CSDH diagnosis, several studies have been reported on the resumption timing of antithrombotic agents after CSDH surgery. Since antithrombotic agents are generally used for other existing medical causes, it is necessary to resumption of anti- thrombotic agents after completion of treatment with CSDH. Phan et al. ${ }^{16)}$ reported, through systematic review and metaanalysis related to the resumption of antithrombotic agents in $\mathrm{CSDH}$, that the most common indication for antithrombotic treatment before onset of CSDH was atrial fibrillation (29.6\%), followed by prosthetic heart valve (16.6\%), recent myocardial infarction (14.1\%), prior stroke or transient ischemic attack (11.6\%), and venous thromboembolism (8.3\%). They also reported that there was no difference in the incidence of hemorrhagic complications and thromboembolic events by comparing early (3-14 days) and late ( $>30$ days) resumption, suggesting that it is feasible to resume early antithrombotic treatment without additional hemorrhagic or thromboembolic risk in selected cases ${ }^{16)}$. In addition, Fornebo et al. ${ }^{7)}$ reported that early ( $<30$ days) resumption of antithrombotic agents are not related to CSDH recurrence and are more beneficial. In this study, because of the limitations of retrospective studies, the timing of antithrombotic agents resumption was not clearly investigated, but in most cases, antithrombotic agents was resumed at least 30 days later.

Although antithrombotic agent subgroups were not included DOACs in this study, studies have recently been conducted on DOACs, which are widely used as a substitute for warfarin. Motoie et al. ${ }^{13)}$ reported that DOACs such as dabigatran, edoxaban, apixaban, and rivaroxaban do not increase the recurrence rate of $\mathrm{CSDH}$. The authors argued that there was no need to hesitate while prescribing antithrombotic agents due to fear of CSDH recurrence. In particular, DOACs have a lower risk of cerebral hemorrhage than the vitamin K antagonists $^{1726)}$. DOACs do not affect the interaction between tissue factor and initial factor VII/VIIa in the extrinsic coagulation pathway unlike the vitamin $\mathrm{K}$ antagonists. Hence, it has been argued that rapid coagulation and reduced hemorrhage expansion can be achieved in cerebral hemorrhage ${ }^{25}$. On the other hand, some studies have argued that these benefits of DOACs differ between intracerebral hemorrhage and extracerebral hemorrhage such as subdural and epidural hemorrhage ${ }^{6,26)}$. Further studies are needed to assess the association between DOACs and CSDH outcomes.

In addition to antithrombotic agents, studies on various other risk factors related to CSDH recurrence have been conducted. Age and gender were not related to CSDH recurrence in the present study, which is consistent with the reports of some studies ${ }^{2,7,8,10,20,21)}$. On the other hand, other studies have 
argued that age and male gender are independent risk factors associated with CSDH recurrence ${ }^{1,3,13,24)}$.

As the present study was conducted retrospectively, only the investigation regarding the use of antithrombotic agents at the time of CSDH diagnosis was performed. Subgroups of antithrombotic agents have been investigated, but did not include patients with DOACs, which have recently increased use. Moreover, although antithrombotic agents are associated with $\mathrm{CSDH}$ recurrence, the finding of this study that antiplatelets and anticoagulants are not relevant suggests that the history related to antithrombotic agents may be a more important factor. However, this study was limited that there are many cases of unclear record to reason for antithrombotic agents taking. In addition, in most cases, the timing of the resumption of antithrombotic agents was different even though it was resumed after the CSDH treatment was completed. And, because of the limitations of retrospective studies, the timing of the antithrombotic agents resumption was not clearly investigated. Several studies reported that early resumption of antithrombotic agents are not related to $\mathrm{CSDH}$ recurrence and are more beneficial, but it may be difficult to generalize the results. The authors plan to conduct further studies on the categorization of antithrombotic agents, and on the optimal timing of resumption of individual subgroups of antithrombotic agents after traumatic brain injury and $\mathrm{CSDH}$.

\section{CONCLUSION}

In the present study, the use of antithrombotic medication at the time of $\mathrm{CSDH}$ diagnosis was observed to be an independent risk factor for CSDH recurrence. Therefore, special attention should be paid to patients with a history of receiving antithrombotic medications at the time of $\mathrm{CSDH}$ diagnosis in the postoperative follow-up of $\mathrm{CSDH}$ patients. Further prospective studies using consistent study design are needed for evaluation of the risk according to each subtype of antithrombotic agents, including DOACs.

\section{CONFLICTS OF INTEREST}

No potential conflict of interest relevant to this article was reported.

\section{INFORMED CONSENT}

This type of study does not require informed consent.

\section{AUTHOR CONTRIBUTIONS}

\author{
Conceptualization : KW \\ Data curation : JC \\ Formal analysis : JC \\ Funding acquisition : $\mathrm{KW}$ \\ Methodology : JP, SC, YK \\ Project administration : JK \\ Visualization : JK \\ Writing - original draft : JC \\ Writing - review \& editing : KW, JC
}

\section{ORCID}

Jongwook Choi https://orcid.org/0000-0003-2593-3870

Jinsu Pyen https://orcid.org/0000-0002-8008-4122

Sungmin Cho https://orcid.org/0000-0002-7593-4815

Jongyeon Kim https://orcid.org/0000-0002-9407-0127

Younmoo Koo https://orcid.org/0000-0002-9054-8250

Kum Whang https://orcid.org/0000-0002-3701-847X

\section{References}

1. Abe Y, Maruyama K, Yokoya S, Noguchi A, Sato E, Nagane M, et al. : Outcomes of chronic subdural hematoma with preexisting comorbidities causing disturbed consciousness. J Neurosurg 126 : 1042-1046, 2017

2. Aspegren $\mathrm{OP}$, Astrand $\mathrm{R}$, Lundgren MI, Romner B : Anticoagulation therapy a risk factor for the development of chronic subdural hematoma. Clin Neurol Neurosurg 115 : 981-984, 2013

3. Borger V, Vatter H, Oszvald Á, Marquardt G, Seifert V, Güresir E : Chronic subdural haematoma in elderly patients: a retrospective analysis of 322 patients between the ages of 65-94 years. Acta Neurochir (Wien) 154 : 1549-1554, 2012

4. Drapkin AJ : Chronic subdural hematoma: Pathophysiological basis for treatment. Br J Neurosurg 5 : 467-473, 1991

5. Echlin FA, Sordillo SV, Garvey TQ Jr : Acute, subacute, and chronic subdural hematoma. J Am Med Assoc 161 : 1345-1350, 1956

6. Fleck RA, Rao LV, Rapaport SI, Varki N : Localization of human tissue factor antigen by immunostaining with monospecific, polyclonal anti- 
human tissue factor antibody. Thromb Res 59 : 421-437, 1990

7. Fornebo I, Sjåvik $K$, Alibeck $M$, Kristiansson $H$, Ståhl F, Förander $P$, et al. : Role of antithrombotic therapy in the risk of hematoma recurrence and thromboembolism after chronic subdural hematoma evacuation: a population-based consecutive cohort study. Acta Neurochir (Wien) 159 : 2045-2052, 2017

8. Fujitani S, Ishikawa O, Miura K, Takeda Y, Goto H, Maeda K : Factors predicting contralateral hematoma growth after unilateral drainage of bilateral chronic subdural hematoma. J Neurosurg 126 : 755-759, 2017

9. Gernsback J, Kolcun JP, Jagid J : To drain or two drains: recurrences in chronic subdural hematomas. World Neurosurg 95 : 447-450, 2016

10. Jung $Y G$, Jung NY, Kim $E$ : Independent predictors for recurrence of chronic subdural hematoma. J Korean Neurosurg Soc 57 : 266-270, 2015

11. Kolias AG, Chari A, Santarius T, Hutchinson PJ : Chronic subdural haematoma: modern management and emerging therapies. Nat Rev Neurol $10: 570-578,2014$

12. Markwalder TM : Chronic subdural hematomas: a review. J Neurosurg $54:$ 637-645, 1981

13. Motoie R, Karashima S, Otsuji R, Ren N, Nagaoka S, Maeda K, et al. : Recurrence in 787 patients with chronic subdural hematoma: retrospective cohort investigation of associated factors including direct oral anticoagulant use. World Neurosurg 118 : e87-e91, 2018

14. Nathan S, Goodarzi Z, Jette N, Gallagher C, Holroyd-Leduc J : Anticoagulant and antiplatelet use in seniors with chronic subdural hematoma: systematic review. Neurology 88 : 1889-1893, 2017

15. Obana WG, Pitts $L H$ : Management of head injury. Extracerebral lesions. Neurosurg Clin N Am 2 : 351-372, 1991

16. Phan K, Abi-Hanna D, Kerferd J, Lu VM, Dmytriw AA, Ho YT, et al. : Resumption of antithrombotic agents in chronic subdural hematoma: a systematic review and meta-analysis. World Neurosurg 109 : e792e799, 2018

17. Phuenpathom N, Choomuang M, Ratanalert S : Outcome and outcome prediction in acute subdural hematoma. Surg Neurol $40: 22-25,1993$

18. Poon MTC, Al-Shahi Salman R : Association between antithrombotic drug use before chronic subdural haematoma and outcome after drainage: a systematic review and meta-analysis. Neurosurg Rev 41 : 439 445, 2018

19. Poon MTC, Rea C, Kolias AG, Brennan PM; British Neurosurgical Trainee Research Collaborative (BNTRC) : Influence of antiplatelet and anticoagulant drug use on outcomes after chronic subdural hematoma drainage. J Neurotrauma, 2019 [Epub ahead of print]

20. Rust T, Kiemer N, Erasmus A : Chronic subdural haematomas and anticoagulation or anti-thrombotic therapy. J Clin Neurosci $13: 823-827$, 2006

21. Tugcu B, Tanriverdi O, Baydin S, Hergunsel B, Günaldı Ö, Ofluoglu E, et al. : Can recurrence of chronic subdural hematoma be predicted? A retrospective analysis of 292 cases. J Neurol Surg A Cent Eur Neurosurg $75: 37-41,2014$

22. Wang $H$, Zhang $M$, Zheng $H$, Xia X, Luo K, Guo F, et al. : The effects of antithrombotic drugs on the recurrence and mortality in patients with chronic subdural hematoma: a meta-analysis. Medicine (Baltimore) 98 : e13972, 2019

23. Wang Y, Zhou J, Fan C, Wang D, Jiao F, Liu B, et al. : Influence of antithrombotic agents on the recurrence of chronic subdural hematomas and the quest about the recommencement of antithrombotic agents: a meta-analysis. J Clin Neurosci 38 : 79-83, 2017

24. Weigel R, Schmiedek P, Krauss JK : Outcome of contemporary surgery for chronic subdural haematoma: evidence based review. J Neurol Neurosurg Psychiatry 74 : 937-943, 2003

25. Wilson D, Charidimou A, Shakeshaft C, Ambler G, White M, Cohen $H$, et al. : Volume and functional outcome of intracerebral hemorrhage according to oral anticoagulant type. Neurology 86 : 360-366, 2016

26. Won SY, Dubinski D, Bruder M, Cattani A, Seifert V, Konczalla J : Acute subdural hematoma in patients on oral anticoagulant therapy: management and outcome. Neurosurg Focus 43 : E12, 2017 